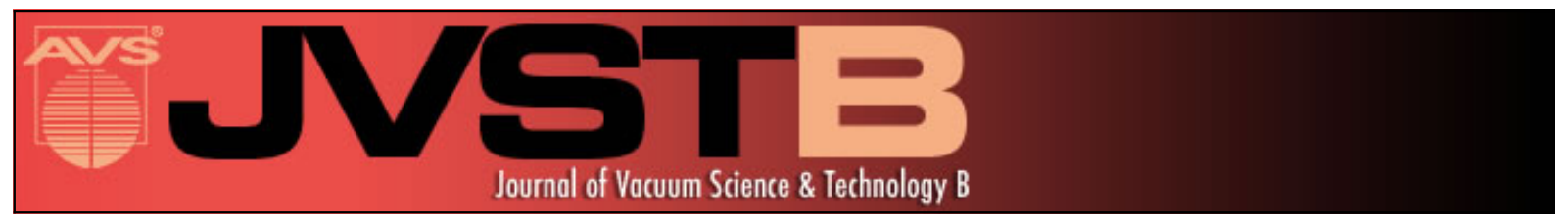

\title{
Reflection high-energy electron diffraction oscillations on rotating substrates
}

W. Braun, H. Möller, S. R. Johnson, and Y.-H. Zhang

Citation: Journal of Vacuum Science \& Technology B 17, 474 (1999); doi: 10.1116/1.590579

View online: http://dx.doi.org/10.1116/1.590579

View Table of Contents: http://scitation.aip.org/content/avs/journal/jvstb/17/2?ver=pdfcov

Published by the AVS: Science \& Technology of Materials, Interfaces, and Processing

\section{Articles you may be interested in}

Phase of reflection high-energy electron diffraction oscillations during (Ba,Sr)O epitaxy on $\mathrm{Si}(100)$ : A marker of Sr barrier integrity

Appl. Phys. Lett. 87, 262905 (2005); 10.1063/1.2158018

Accurate growth rate determination on rotating substrates using electron diffraction dynamics

Appl. Phys. Lett. 74, 138 (1999); 10.1063/1.122975

New model for reflection high-energy electron diffraction intensity oscillations

J. Vac. Sci. Technol. B 16, 2404 (1998); 10.1116/1.590182

Reflection high-energy electron diffraction during substrate rotation: A new dimension for in situ characterization J. Vac. Sci. Technol. B 16, 1507 (1998); 10.1116/1.589976

Reflection high-energy electron diffraction oscillations during growth of GaAs at low temperatures under high As overpressure

Appl. Phys. Lett. 71, 1540 (1997); 10.1063/1.119973

\section{Instruments for Advanced Science}

Contact Hiden Analytical for further details: W www.HidenAnalytical.com E info@hiden.co.uk CLICK TO VIEW our product catalogue

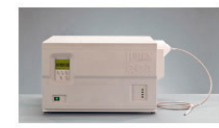

Gas Analysis

dynamic measurement of reaction gas streams catalysis and thermal analysis

molecular beam studies

fermentation, environmental and ecological studies

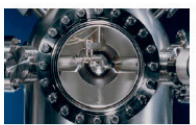

Surface Science , UHVTPD SIMS

end point detection in ion beam etch elementa limaging - surface mapoing

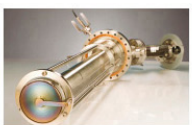

Plasma Diagnostics plasma source characterization etch and deposition process reaction kinetic studies r analysis of neutral and radical species

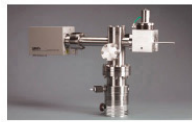

Vacuum Analysis i partial pressure measurement and control of process gases reactive sputter process contro vacuum diagnostics vacuum coating process monitoring 


\title{
Reflection high-energy electron diffraction oscillations on rotating substrates
}

\author{
W. Braun ${ }^{\mathrm{a})}$ \\ Center for Solid State Electronics Research and Department of Electrical Engineering, \\ Arizona State University, Tempe, Arizona 85287-6206 \\ H. Möller \\ Fraunhofer-Insitut für Integrierte Schaltungen-A, D-91058 Erlangen, Germany
}

S. R. Johnson and Y.-H. Zhang

Center for Solid State Electronics Research and Department of Electrical Engineering, Arizona State University, Tempe, Arizona 85287-6206

(Received 22 October 1998; accepted 18 December 1998)

\begin{abstract}
We compare different methods to measure reflection high-energy electron diffraction oscillations on rotating substrates. The best signal-to-noise ratio as well as the highest accuracy is obtained by measuring the full width at half maximum of the specular spot perpendicular to the surface. The accuracy of the method is well within $1 \%$ and offers a practical way to accurately determine growth rates for device fabrication. (c) 1999 American Vacuum Society. [S0734-211X(99)04502-3]
\end{abstract}

\section{INTRODUCTION}

Although reflection high-energy electron diffraction (RHEED) has always been a popular characterization method in molecular beam epitaxy (MBE), ${ }^{1}$ the number of studies investigating RHEED with substrate rotation is limited. This is mainly due to the high noise levels introduced by mechanical vibrations and the periodic modulation of the signal with the rotation frequency and its harmonics. ${ }^{2}$ Whereas azimuthal scanning techniques ${ }^{3}$ allow access to almost the entire upper half of reciprocal space, the data in these measurement modes are updated only once per revolution. Azimuthal scans are therefore not suitable for the measurement of growth oscillations due to the layer-by-layer growth of the crystal. The only part of the diffraction pattern that is visible continuously during rotation is the (00) rod. The specularly reflected spot moves up and down along this rod during rotation depending on substrate wobble and miscut. Its intensity typically varies about three orders of magnitude during one rotation for a nongrowing surface. ${ }^{4}$ In addition, both the phase and the amplitude of RHEED intensity oscillations are strong functions of the azimuthal ${ }^{5}$ and polar $^{6-8}$ angles of the electron beam. This leads to ill-defined growth frequencies and beating in intensity oscillation measurements.

In one approach, the substrate is therefore rotated at high speeds, resulting in an averaging of these variations on a timescale much smaller than the growth oscillation period. ${ }^{9}$ This method works well from a diffraction point of view, but leads to excessive wear of the rotation drive and has not been widely adopted. A different method uses a spot tracking algorithm combined with numerical filtering techniques to determine the specular spot size and measure its variations during growth. ${ }^{10}$ The signal in this case is quite noisy and a narrow filter window needs to be applied to determine the

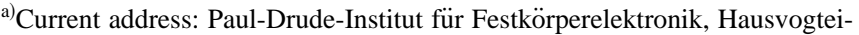
platz 5-7, D-10117 Berlin, Germany; electronic mail: braun@pdi-berlin.de growth frequency, which means that the growth frequency has to be known quite accurately before the measurement. The use of the specular spot size or full width at half maximum (FWHM), though, is advantageous, since this signal typically varies by not more than one order of magnitude, allowing simple and fast processing of the digitized data. Also, the spot size does not vary as much as its intensity with changing diffraction conditions for a given static surface.

In this article, we extend this approach by making use of the strongly anisotropic sensitivity of RHEED parallel and perpendicular to the beam along the surface. Instead of measuring the total spot size, we study its FWHM perpendicular to the surface along the (00) beam. Due to the small angle the Ewald sphere makes with the (00) rod, the resulting signal is already amplified by approximately a factor of $\cot \theta$, where $\theta$ is the angle the electron beam makes with the sample surface. This greatly reduces the noise levels. In addition, the FWHM algorithm already compensates for substrate miscut and wobble without having to resort to twodimensional spot tracking algorithms. Finally, phase-locked substrate rotation results in improved accuracy through the inclusion of satellite peaks in the measurement.

\section{EXPERIMENTS AND DISCUSSION}

The experiments were performed in a VG V80 MBE system using the standard right angle substrate manipulator and 2 in. GaAs (001) substrates. The substrate rotation motor was replaced by a phase-locked dc motor synchronized to the timebase the RHEED charge coupled device (CCD) camera was using. The CCD camera and the PC-based data acquisition system ${ }^{11}$ allowed an acquisition rate of $50 \mathrm{~Hz}$, twice that of a standard video frame rate. The RHEED gun was the standard VG type. For the measurements during rotation, the RHEED intensities were measured along a line perpendicular to the substrate surface through the specular spot.

Figure 1 shows a comparison of the data from three different measurement methods. The top line represents the tra- 


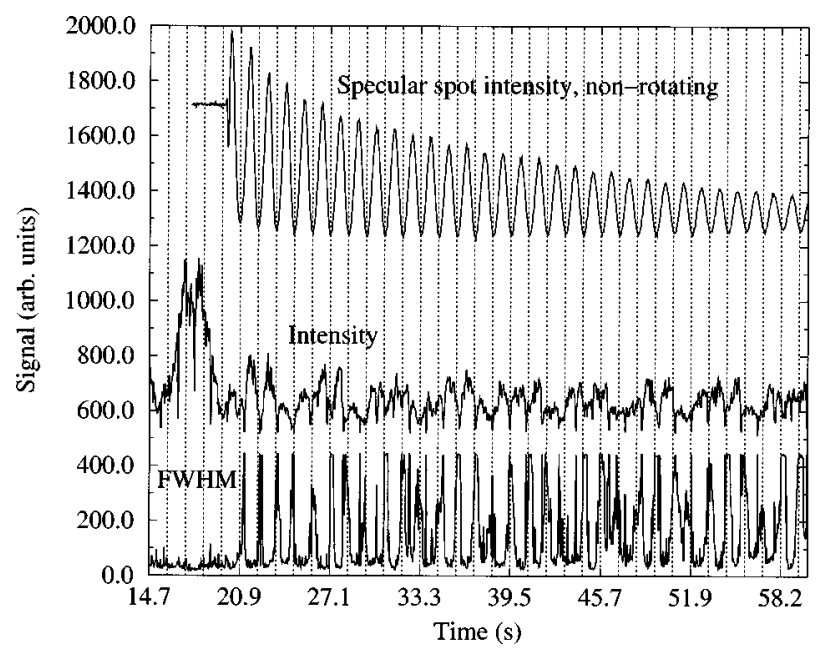

FIG. 1. GaAs-on-GaAs RHEED oscillation signals obtained by three different measurement methods for identical growth conditions. Top curve: Integrated specular spot intensity, nonrotating; center curve: integrated intensity from measurement line along the (00) streak, rotating; bottom curve: FWHM of the specular spot along (00), rotating.

ditional measurement of the integrated spot intensity during growth without substrate rotation. The center and bottom traces were processed from the same linescan along the (00) streak. In the center curve, the intensity along the line was integrated, whereas the bottom curve shows the FWHM along the line. Compared to the signals obtained during rotation, the top trace shows stronger damping. The difference in the damping rates can be explained by variations of the growth rate along the probed surface area in nonrotating growth. ${ }^{12}$ This type of damping should be reduced during rotation since the growth rate is averaged across the wafer. For the integrated intensity during rotation, however, the noise level also increases strongly, resulting in about the same number of usable oscillations until the signal vanishes in the noise. The FWHM signal, on the other hand, shows a good signal-to-noise ratio, and the crossover between signal and noise is reached after typically twice the number of oscillations compared to the nonrotating intensity signal.

Another important factor for the measurements on rotating substrates is the suppression of the rotation frequency in the signal. The dashed lines in Fig. 1 indicate the period of the growth oscillations. The fourfold rotational symmetry of the cubic crystal structure underlying the GaAs (001) surface results in a modulation of the rotating intensity signal (center curve), coinciding with the growth frequency roughly every 11 growth oscillations. This component with a slightly higher frequency is also present in the FWHM signal, but in contrast to the intensity data there is also a strong peak at each marker line. In the intensity measurement, minima at the dashed line positions are weak if they do not coincide with the rotation minima. This means that the suppression of the rotation frequency and its harmonics is stronger in the FWHM measurement.

The difference can be more clearly visualized in frequency space. The Fourier transform of the measurements in Fig. 1 are shown in Fig. 2. The solid line represents the

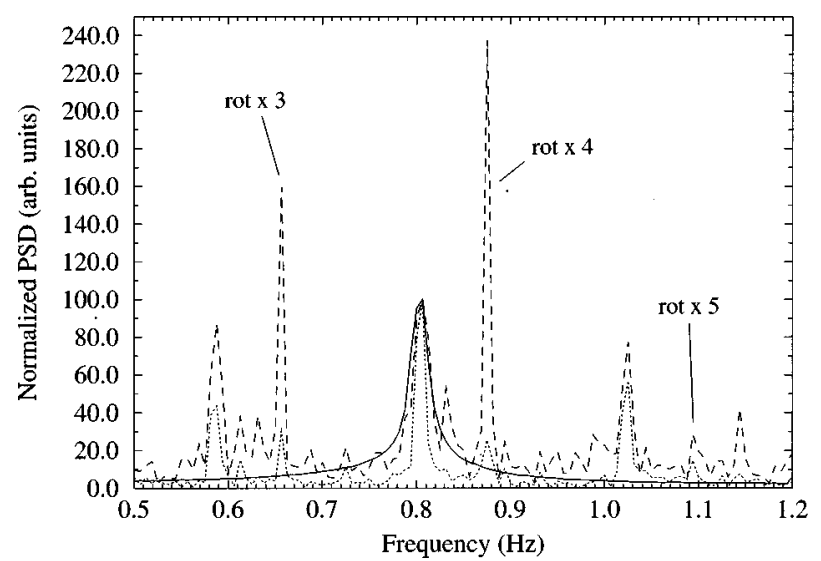

FIG. 2. Comparison of the Fourier spectra (power spectral density, PSD) obtained by transforming the three signals in Fig. 1. The results are normalized to the growth frequency peak. The solid line corresponds to the nonrotating integrated specular spot intensity, the dashed line to the integrated line intensity during rotation, and the dotted line to the FWHM of the specular spot along (00).

nonrotating intensity signal, the dashed line is from the rotating intensity signal, and the dotted line is the transform of the rotating FWHM signal. All traces are normalized to the magnitude of the growth oscillation peak maximum. The fourfold rotation frequency peak is 10 times stronger for the intensity measurement during rotation compared to that of the FWHM measurement. In addition to the strong rotation frequency suppression, the FWHM measurement also has the best frequency resolution, measured by the FWHM of the growth oscillation peak. The values are $0.027 \mathrm{~Hz}$ for nonrotating intensity, $0.021 \mathrm{~Hz}$ for rotating intensity, and $0.013 \mathrm{~Hz}$ for rotating FWHM. The width of this peak is affected by damping and noise of the time space signal and by the duration of the measurement. The measurement time for all three curves was identical. The broadening of the nonrotating intensity peak is mainly due to the strong damping of the signal. ${ }^{13}$ Based on the present data, we cannot unambigu-

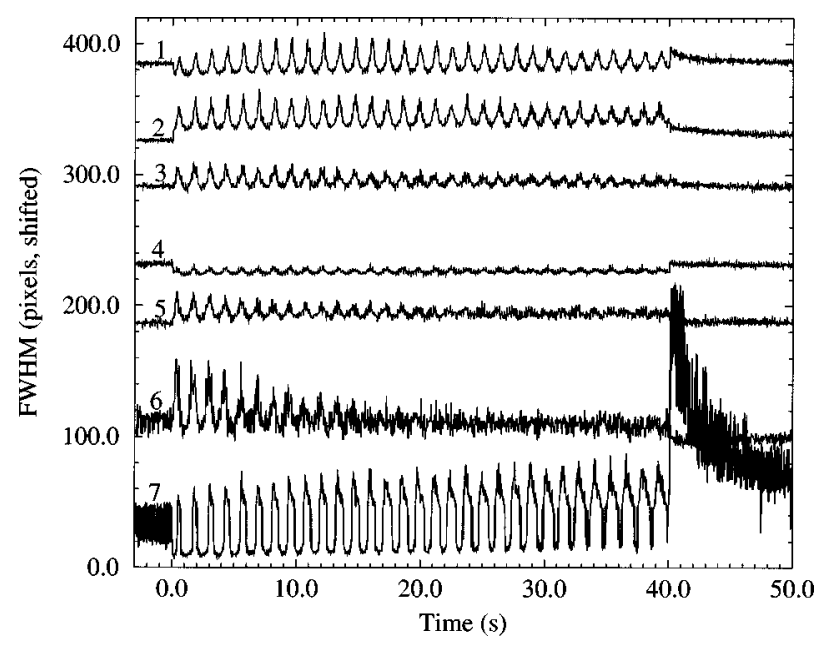

FIG. 3. FWHM signals taken perpendicular and parallel to the substrate surface at different positions along the streak. The substrate is not rotating. The positions of the measurement lines are shown in the inset of Fig. 4. 


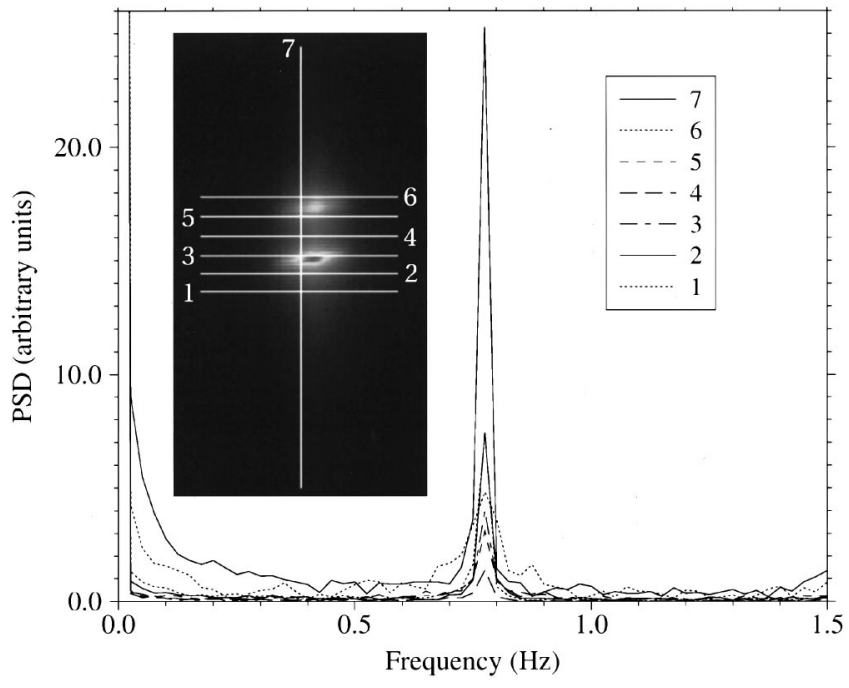

FIG. 4. Comparison of the rotation frequency peak magnitudes for the different FWHM signals measured along the lines given in the inset. The measurement perpendicular to the surface (trace 7) results in the largest peak.

ously decide whether the difference between the two rotating signal peaks is due to the different noise levels or due to differences in damping.

The accuracy of the rotating measurement modes can be further increased by using phase-locked substrate rotation. This means that each substrate rotation corresponds exactly to a certain number of measurement points. The satellite peaks to the growth frequency are then spaced at an exactly known distance and can be included in the position determination as well, thereby increasing the accuracy of the measurement. ${ }^{14}$ For long measurements, accuracies within $0.5 \%$ can be achieved.

To study the dependence of the FWHM measurement on the measurement direction across the specular spot, FWHM measurements of GaAs homoepitaxy were performed parallel and perpendicular to the surface on a nonrotating substrate. The time-space results are shown in Fig. 3. The geometry of the measurement lines as well as the frequencyspace results are shown in Fig. 4. Since the Ga shutter was magnetically coupled, the specular spot moved left during growth, as seen in the inset of Fig. 4, resulting in large noise levels before and after growth in trace 7 .

The results in Fig. 3, plotted on the same scale, clearly show the intrinsic amplification of the signal measured perpendicular to the substrate surface. In addition, the signals parallel to the surface are more strongly damped, combined with an initial increase for curves 1 and 2. These amplitude variations should broaden the frequency peaks. The short measurement time of $40 \mathrm{~s}$, however, results in uncertaintyrelation limited peak widths in Fig. 4 and, with the exception of line 6 , these differences cannot be resolved. It is clear, however, that the absolute noise levels for the different measurement directions are comparable and therefore the signalto-noise ratio of curve 7 is better due to its larger absolute signal, even despite the fact that the spot is split in this direction.

In conclusion, we have demonstrated that the measurement of the specular spot width perpendicular to the surface allows growth oscillation frequency determination on rotating substrates with dramatically increased accuracy. This is due to a strong suppression of rotation-related frequency contributions, an intrinsic signal amplification due to the scattering geometry, and a resulting increase of the signal-tonoise ratio.

\section{ACKNOWLEDGMENT}

Part of this work was supported by a DARPA program (Contract No. MDA972-95-1-0016) managed by G. Pomrenke.

${ }^{1}$ Reflection High-Energy Electron Diffraction and Reflection Electron Imaging of Surfaces, edited by P. K. Larsen and P. J. Dobson, NATO ASI Ser. 188 (Plenum, New York, 1988).

${ }^{2}$ G. W. Turner and A. J. Isles, J. Vac. Sci. Technol. B 10, 1784 (1992).

${ }^{3}$ W. Braun, H. Möller, and Y.-H. Zhang, J. Vac. Sci. Technol. B 16, 1507 (1998).

${ }^{4}$ Z. Mitura and P. Maksym, Phys. Rev. Lett. 70, 2904 (1993), and references therein.

${ }^{5}$ J. Resh, K. D. Jamison, J. Strozier, A. Bensaoula, and A. Ignatiev, Phys. Rev. B 40, 11799 (1989).

${ }^{6}$ J. Zhang, J. H. Neave, P. J. Dobson, and B. A. Joyce, Appl. Phys. A: Solids Surf. 42, 317 (1987).

${ }^{7}$ W. Braun, L. Däweritz, and K. H. Ploog, Phys. Rev. Lett. 80, 4935 (1998).

${ }^{8}$ W. Braun, L. Däweritz, and K. H. Ploog, J. Vac. Sci. Technol. B 16, 2404 (1998).

${ }^{9}$ J. P. A. van der Wagt and J. S. Harris, Jr., J. Vac. Sci. Technol. B 12, 1236 (1992).

${ }^{10}$ D. A. Collins, G. O. Papa, and T. C. McGill, J. Vac. Sci. Technol. B 13, 1953 (1995).

${ }^{11}$ SAFIRE, VTS Schwarz/FhG Erlangen, vts.js@t-online.de.

${ }^{12}$ J. P. A. van der Wagt, K. L. Bacher, G. S. Solomon, and J. S. Harris, Jr., J. Vac. Sci. Technol. B 10, 825 (1992).

${ }^{13}$ J. E. Cunningham, R. N. Pathak, and W. Y. Jan, Appl. Phys. Lett. 68, 394 (1996).

${ }^{14}$ W. Braun, H. Möller, and Y.-H. Zhang, Appl. Phys. Lett. (to be published). 